\title{
Exposición al humo de tabaco en hogares de la Ciudad de México: análisis de nicotina ambiental y en cabello de niños y mujeres
}

\author{
Tonatiuh Barrientos-Gutiérrez, MD, M en C, ${ }^{(1,2)}$ Luz Myriam Reynales-Shigematsu, MD, M en C, Dr en C, ${ }^{(2)}$ \\ Erika Ávila-Tang, M en C, PhD, ${ }^{(3)}$ Heather Wipfli, MA, ${ }^{(3)}$ Eduardo Lazcano-Ponce, MD, M en C, Dr en C. ${ }^{(2)}$
}

\begin{abstract}
Barrientos-Gutiérrez T, Reynales-Shigematsu LM, Ávila-Tang E,Wipfli H, Lazcano-Ponce E. Exposición al humo de tabaco en hogares de la Ciudad de México: análisis de nicotina ambiental y en cabello de niños y mujeres.

Salud Publica Mex 2007;49 supl 2:S224-S232.
\end{abstract}

\section{Resumen}

Objetivo. En México no se han efectuado evaluaciones de la exposición al humo de tabaco ambiental en los hogares ni en sus habitantes. El objetivo de este estudio es cuantificar los niveles de nicotina ambiental en hogares de la Ciudad de México, evaluando simultáneamente los niveles de nicotina en el cabello de niños y mujeres. Material y métodos. En julio de 2005 se seleccionaron 4I hogares de la Ciudad de México a conveniencia, $20 \%$ sin fumadores y $80 \%$ con fumadores. Se colocaron monitores pasivos de nicotina en los hogares por una semana y se obtuvieron muestras de cabello de mujeres no fumadoras y niños para cuantificar nicotina. Las muestras fueron enviadas a la Universidad Johns Hopkins, donde la nicotina se extrajo y analizó con cromatografía de gases. De manera adicional, se aplicaron encuestas de opinión y conductas relacionadas con el humo de tabaco ambiental. Resultados. Las concentraciones de nicotina ambiental tuvieron una mediana de $0.08 \mu \mathrm{g} / \mathrm{m}^{3}$ (RIC 0.0 I-0.64), en el cabello de los niños $0.05 \mathrm{ng} / \mathrm{mg}$ (RIC 0.05-0.29) y en el cabello de las mujeres $0.05 \mathrm{ng} / \mathrm{mg}$ (RIC 0.05-0.19). Las concentraciones de nicotina ambiental y en el cabello de los niños mostraron una correlación alta $\left(r_{s}=0.49\right)$, y se incrementó con el número de fumadores en el hogar. La mayoría de los encuestados apoya las medidas de control del humo de tabaco ambiental. Conclusiones. Se observaron grados particularmente altos

\author{
Barrientos-Gutiérrez T, Reynales-Shigematsu LM, \\ Ávila-Tang E,Wipfli H, Lazcano-Ponce E. \\ Environmental tobacco smoke exposure in homes \\ of Mexico City: analysis of environmental samples \\ and children and women hair. \\ Salud Publica Mex 2007;49 suppl 2:S224-S232.
}

\section{Abstract}

Objective. In Mexico no evaluation of environmental tobacco smoke exposure in homes or habitants has been conducted. The objective of this study is to quantify environmental nicotine in Mexico City homes, simultaneously evaluating nicotine levels in children and women. Material and Methods. In July 2005 a convenience sample of 4 I homes was selected, $20 \%$ without smokers, $80 \%$ with smokers. Nicotine passive monitors were allocated in homes for one week, obtaining samples of hair from inhabitant non-smoking women and children.Samples were taken to the Johns Hopkins University where nicotine was extracted and analyzed using gas chromatography. A survey of opinions and behaviors related to environmental tobacco smoke was conducted. Results. Environmental nicotine concentrations had a median of 0.08 $\mu \mathrm{g} / \mathrm{m} 3$ (IQR 0.0I-0.64), in children's hair $0.05 \mathrm{ng} / \mathrm{mg}$ (IQR $0.05-0.29$ ), and in women's hair $0.05 \mathrm{ng} / \mathrm{mg}$ (IQR 0.05-0.19). Environmental nicotine concentrations and in children's hair were highly correlated $\left(r_{s}=0.49\right)$, and increased with the number of smokers at home. The majority of adults surveyed showed support towards measures of environmental tobacco smoke control. Conclusions. Homes are important spaces of exposure to environmental tobacco smoke. Particularly high levels of exposure were observed in children's hair, attributable to the presence of environmental nicotine at home.

\footnotetext{
(I) The University of Texas, School of Public Health. EUA.

(2) Instituto Nacional de Salud Pública. México.
}

(3) Johns Hopkins Bloomberg School of Public Health. EUA.

Fecha de recibido: 4 de abril de 2007 - Fecha de aprobado: 30 de abril de 2007 Solicitud de sobretiros: Mtro.Tonatiuh Barrientos-Gutiérrez. The Southwest Center For Occupational and Environmental Health The University of Texas, School of Public Health at Houston. I 200 Hermann Pressler, w- I004-I Houston, Texas, 77030, USA.

Correo electrónico: tonatiuh.barrientos@uth.tmc.edu 
de exposición en niños atribuibles a la presencia de nicotina ambiental en el hogar. Deben implementarse acciones preventivas integrales para eliminar el tabaquismo activo y evitar el consumo de tabaco en el hogar.

Palabras clave: humo de tabaco; nicotina; aire; cabello; hogares; mujeres; niños; México
Integral preventive activities to eliminate active smoking and to avoid tobacco consumption at home are required.

Keywords: tobacco smoke; nicotine; air; hair; homes; children; women; Mexico
$E^{\prime}$ humo de tabaco ambiental es una mezcla de gases y part culas derivadas de la combustión del tabaco con al menos 250 compuestos tóxicos o carcinógenos. ${ }^{1}$ La exposición involuntaria ha sido identificada como una causa importante de enfermedades respiratorias, cardiovasculares y de diferentes tipos de cánceres en adultos. ${ }^{1}$ Se ha estimado que las mujeres no fumadoras casadas con fumadores tienen $24 \%$ de exceso de riesgo de padecer cáncer de pulmón ${ }^{2}$ y $30 \%$ de padecer enfermedad coronaria. ${ }^{1}$ En niños, la exposición al humo de tabaco ambiental ha sido asociada con el s ndrome de muerte súbita del neonato, bajo peso al nacer, asma y otras enfermedades respiratorias, enfermedades del o do medio, reducción de la función pulmonar, cáncer y mayor deterioro de enfermedad cardiovascular previa. ${ }^{1}$

La acumulación de evidencia que vincula el humo de tabaco ambiental con efectos nocivos para la salud ha estimulado la investigación sobre la exposición involuntaria al humo de tabaco ambiental en lugares públicos $^{3,4}$ e impulsado leyes y normativas para su eliminación. ${ }^{5}$ En los pa ses que han logrado prohibir el consumo de tabaco en lugares públicos se observa una reducción efectiva de la exposición y sus efectos, ${ }^{6-9}$ lo que ha servido para alentar el desarrollo de esfuerzos similares en el ámbito privado, como el hogar. El hogar es un espacio de exposición de particular importancia para niños y mujeres no fumadoras que conviven con fumadores. ${ }^{10}$ El control de la exposición al humo de tabaco en el hogar representa un reto, ya que el hogar es menos susceptible al control externo derivado de la legislación, y, por lo tanto, requiere la participación voluntaria de los fumadores.

A través de programas integrales, Estados Unidos, ${ }^{11}$ Reino Unido ${ }^{12}$ y Nueva Zelanda ${ }^{13}$ incrementaron la prevalencia de hogares libres de humo de tabaco. Los mejores resultados provienen del control de precios al tabaco, programas educativos, espacios públicos libres de humo de tabaco y programas de apoyo para dejar de fumar. ${ }^{14}$ La Organización Mundial para la Salud y la Organización Panamericana de la Salud consideran prioritaria la investigación de la exposición al humo de tabaco ambiental en hogares de pa ses en desarrollo, con miras a impulsar propuestas para su eliminación. ${ }^{15,16}$ En México, la alta prevalencia de tabaquismo activo $(34.8 \%$ ) y la alta exposición de la población infantil al humo de tabaco $(45.5 \%)^{17}$ hacen necesaria una evaluación sistemática.

El presente estudio tuvo como objetivo cuantificar los niveles de exposición al humo de tabaco ambiental en hogares de fumadores y no fumadores de la Ciudad de México, con evaluación simultánea del impacto personal de la exposición en niños y mujeres no fumadoras. Un segundo objetivo fue explorar las opiniones y comportamientos relacionados con la generación y control de la exposición al humo de tabaco ambiental.

\section{Material y métodos}

El presente estudio se basó en un protocolo de investigación desarrollado por la Universidad Johns Hopkins, aplicado simultáneamente en otros 29 pa ses en desarrollo con la finalidad de proveer información que ayude a desarrollar iniciativas para eliminar la exposición al humo de tabaco ambiental en el hogar.

\section{Selección de hogares}

En julio de 2005, se seleccionó por conveniencia una muestra de 41 hogares con base en el marco muestral de la delegación Tlalpan desarrollado con anticipación por el Instituto Nacional de Salud Pública. Para ser incluidos en el estudio, los hogares deb an contar con al menos un niño menor de 10 años; $80 \%$ con al menos un fumador, y en el restante $20 \%$ no deb an vivir fumadores. Todos los participantes, adultos y niños, recibieron información detallada sobre los procedimientos del estudio y proporcionaron un consentimiento informado. El protocolo de estudio fue aprobado por el comité de ética de la Universidad Johns Hopkins y por el del Instituto Nacional de Salud Pública.

\section{Cuantificación de la exposición al humo de tabaco ambiental}

Para la evaluación se cuantificó la nicotina en fase de vapor mediante monitores de difusión pasiva de nicotina. Los monitores de difusión pasiva consisten en un 
caset de plástico que contiene un filtro impregnado en bisulfato de sodio, un compuesto qu mico con alta afinidad por la nicotina, ${ }^{18} \mathrm{y}$ cuentan con amplia validación y el respaldo de múltiples empleos en estudios de cuantificación del humo de tabaco ambiental. ${ }^{19}$ En cada hogar se colocó un monitor a uno o dos metros del suelo durante una semana en el área donde las personas pasaban la mayor parte del tiempo, evitando los espacios con poca circulación de aire como rincones o detrás de las cortinas. Para efectos de control de calidad se incluyeron $10 \%$ de monitores duplicados y $20 \%$ de monitores blancos. En 10\% de los hogares se llevó a cabo una visita a mitad del per odo de monitoreo para verificar la adecuada colocación de los monitores. $\mathrm{Al}$ concluir el monitoreo, los dispositivos fueron recolectados y enviados al laboratorio de nicotina de la Universidad Johns Hopkins, donde fueron analizados mediante cromatograf a gaseosa con detección selectiva de nitrógeno. Las concentraciones de nicotina para cada filtro fueron calculadas dividiendo la cantidad de nicotina encontrada en el filtro entre el volumen de aire muestreado (tasa de flujo $24 \mathrm{ml} / \mathrm{min}$ ).$^{18}$ Las concentraciones $\mathrm{m}$ nimas de detección (CMD) de nicotina fueron calculadas a partir de la curva de regresión y ajustadas por el periodo de muestreo. En el presente estudio, tres casas tuvieron valores de nicotina debajo del nivel $\mathrm{m}$ nimo de detección $\left(0.003 \mu \mathrm{g} / \mathrm{m}^{3}\right)$, por lo cual se les asignó el valor de la mitad de la concentración $m$ nima de detección.

\section{Cuantificación de nicotina en cabello}

Para la evaluación personal de la exposición al humo de tabaco ambiental se obtuvieron muestras de cabello de niños y mujeres no fumadoras en todos los hogares reclutados para el estudio. La determinación de nicotina en cabello es una opción promisoria para evaluar la exposición al humo de tabaco ambiental de más larga data, ya que cuantifica los valores de nicotina que quedaron atrapados en la matriz del cabello durante su crecimiento natural, a un ritmo de $1.1 \mathrm{~cm} / \mathrm{mes}$. Hasta el momento la determinación de nicotina en cabello ha permitido diferenciar fumadores y no fumadores, as como personas que manifiestan exposición o no a humo de tabaco ambiental. ${ }^{20}$ Para el presente estudio se utilizó una técnica estandarizada por la Universidad Johns Hopkins para la recolección y análisis de muestras. En cada hogar se solicitó la participación de al menos un niño y una mujer no fumadora para proveer la muestra de cabello el mismo $d$ a en que se colocaron los monitores de nicotina. Para la toma de la muestra, un miembro del equipo de investigación seleccionó un mechón de 30 a 50 cabellos de la parte posterior de la cabeza, al que cortó lo más cerca de la ra zy asegurando una longitud no mayor a $12 \mathrm{~cm}$ medidos de la ra $\mathrm{z}$ a la punta. De manera adicional, se recabó información sobre tratamientos al cabello y la fecha en que fueron llevados a cabo. El cabello cortado se colocó en una bolsa con cierre hermético y se envió al laboratorio de nicotina de la Universidad Johns Hopkins, donde cada muestra de cabello fue recortada a tres cent metros, medidos de la ra $\mathrm{z}$ a la punta. Fueron seleccionados 30 miligramos de cabello para extraer la nicotina con el método de dilución isotópica y un estándar interno, y la nicotina se cuantificó en un cromatógrafo de gases. Las concentraciones se estimaron dividiendo la cantidad de nicotina encontrada (ng) entre el peso original de la muestra $(\mathrm{mg})$.

\section{Opiniones y conductas relacionadas con} el humo de tabaco ambiental

Se administró una encuesta de opinión y conductas relacionadas con la exposición al humo de tabaco ambiental a las mujeres que proporcionaron la muestra de cabello y a otro ocupante de la casa, de preferencia el padre del niño o niña. El cuestionario recolectó información sobre caracter sticas demográficas de los integrantes del hogar, consumo activo de tabaco del encuestado, su cónyuge y otros habitantes del hogar; exposición al humo de tabaco ambiental en el hogar y en lugares públicos y opiniones sobre la exposición al humo de tabaco ambiental y las pol ticas de control en el hogar y los lugares públicos.

\section{Análisis}

Para la descripción de las concentraciones de nicotina ambiental, se calcularon la mediana, el rango intercuart lico y los valores máximos y m nimos de todos los hogares, y se dispusieron estratos de acuerdo con el número de fumadores en cada hogar. Para las concentraciones en cabello, se calcularon los mismos estimadores dividiendo la muestra en mujeres no fumadoras y niños, y estratificando por el número de fumadores en el hogar. Una prueba de tendencia para comprobar la relación entre el número de fumadores y las concentraciones de nicotina ambiental y en cabello se obtuvo ajustando un modelo de regresión lineal con el logaritmo natural de las concentraciones de nicotina como variable dependiente y el número de fumadores en el hogar como variable independiente. Dada la falta de normalidad de los datos para la correlación de las concentraciones de nicotina en ambiente y en cabello, se utilizó la correlación de Spearman. Todos los análisis fueron llevados a cabo en Stata 8.2. 


\section{Resultados}

El cuadro I muestra la distribución de los hogares y los sujetos participantes en el estudio. La mayor a de los niños $(70 \%)$ pertenec a a edades comprendidas entre los 6 y los 10 años. La mayor a de las mujeres que proveyeron muestra de cabello se encontraba entre los $17 \mathrm{y}$ los 35 años (57\%), hab a estudiado hasta secundaria $(67 \%)$ y era ama de casa o se encontraba sin empleo $(78.6 \%)$. La mayor a de los hombres fumadores contaba con educación hasta el nivel de secundaria (59\%) y estaba sin empleo remunerado $(80 \%)$.

El cuadro II presenta las concentraciones de nicotina ambiental en los hogares y en el cabello de mujeres no fumadoras y niños. El 93\% de los monitores colocados en los hogares estuvo por encima de las CMD (0.003 $\left.\mu \mathrm{g} / \mathrm{m}^{3}\right)$. Las concentraciones de nicotina ambiental presentaron una mediana de $0.08 \mu \mathrm{g} / \mathrm{m}^{3}$ con rango intercuart lico (RIC) entre 0.01 y $0.64 \mu \mathrm{g} / \mathrm{m}^{3}$. Los valores de nicotina aumentan conforme se incrementa el número de fumadores en el hogar, y esta relación es estad sticamente significativa $(p=0.032)$. El 53\% de las muestras de cabello de niños presentó concentraciones de nicotina por encima de los niveles $\mathrm{m}$ nimos de detección $(0.054$ $\mathrm{ng} / \mathrm{mg}$ ), con una mediana de $0.05 \mathrm{ng} / \mathrm{mg}$ (RIC 0.05-0.29 $\mathrm{ng} / \mathrm{mg})$. Al estratificar por el número de fumadores en el hogar pudo observarse que las concentraciones de nicotina en cabello de los niños aumentan significativamente $(p=0.001)$ conforme se incrementa el número de fumadores en el hogar (ver figura 1). El $43 \%$ de las muestras de cabello de mujeres tuvo concentraciones por encima del 1 mite de detección (0.054 ng/mg). Las concentraciones de nicotina en el cabello de las mujeres no fumadoras presentó una mediana de $0.05 \mathrm{ng} / \mathrm{mg}$ (RIC 0.05-0.19 ng/mg). El análisis estratificado no reveló una tendencia clara. Las correlaciones entre nicotina ambiental y nicotina en cabello fueron distintas para mujeres y niños. En el caso de las mujeres, la correlación de Spearman entre la nicotina ambiental y la nicotina encontrada en el cabello fue de $0.04(p=0.79)$, mientras que para los niños fue de $0.49(p<0.001)$.

Los factores de exposición y generación de humo de tabaco ambiental dentro y fuera de los hogares se presentan en el cuadro III. Treinta y cinco por ciento de las mujeres no fumadoras encuestadas manifestó que su cónyuge fumaba dentro del hogar, $72.5 \%$ informó exposición al humo de tabaco ambiental en los medios de transporte, $52.5 \%$ en lugares públicos y $37.5 \%$ en restaurantes. Ochenta y tres por ciento de los fumadores encuestados reconoció fumar frente a los niños, mientras que $74 \%$ aceptó que fumaba dentro del hogar. Las opiniones respecto al humo de tabaco ambiental de los adultos encuestados se resumen en la figura 2. Más

\section{Cuadro I}

Características de hogares y habitantes en estudio. Ciudad de México, Julio de 2005

\begin{tabular}{|c|c|c|}
\hline Variables & Frecuencia & Porcentaje* \\
\hline \multicolumn{3}{|l|}{ Casas $(n=4 I)$} \\
\hline \multicolumn{3}{|c|}{ Tipo de construcción } \\
\hline Casa aislada & 30 & 73.2 \\
\hline Dúplex & 10 & 24.4 \\
\hline Condominio & 1 & 2.4 \\
\hline \multicolumn{3}{|c|}{ Número de fumadores en casa } \\
\hline 0 & 8 & 19.5 \\
\hline 1 & 24 & 58.5 \\
\hline 2 y más & 9 & 22.0 \\
\hline \multicolumn{3}{|l|}{ Niños $(n=55)$} \\
\hline \multicolumn{3}{|l|}{ Edad } \\
\hline $0-5$ años & 17 & 30.9 \\
\hline 6-10 años & 28 & 69.1 \\
\hline
\end{tabular}

Mujeres no fumadoras ${ }^{\ddagger}(n=42)$

Edad

\begin{tabular}{lcc} 
I7-35 años & 24 & 57.1 \\
\hline $36-55$ años & 14 & 33.3 \\
\hline$>55$ años & 2 & 4.8 \\
\hline $\begin{array}{l}\text { Escolaridad } \\
\text { Primaria }\end{array}$ & 7 & 16.7 \\
\hline Secundaria & 28 & 66.7 \\
\hline Bachillerato & 5 & 11.9 \\
\hline $\begin{array}{l}\text { Condición de empleo } \\
\text { Desempleada }\end{array}$ & 33 & 78.6 \\
\hline Empleada & 7 & 16.7
\end{tabular}

\begin{tabular}{lll}
\hline Tratamiento en el cabello & 7 & \\
Sí & 24 & 57.1 \\
\hline No & 18 & 42.9 \\
\hline
\end{tabular}

Cohabitantes $^{\S}(n=44)$

Género

\begin{tabular}{lcc} 
Masculino & 38 & 86.4 \\
\hline Femenino & 6 & 13.6 \\
\hline $\begin{array}{l}\text { Edad } \\
\text { I7-35 años }\end{array}$ & 20 & 45.5 \\
\hline 36-55 años & 20 & 45.5 \\
\hline$>55$ años & 4 & 9.1 \\
\hline $\begin{array}{l}\text { Escolaridad } \\
\text { Primaria }\end{array}$ & \\
\hline Secundaria & 11 & 25.0 \\
\hline Bachillerato o mayor & 26 & 59.1 \\
\hline ¿Fumó en los últimos 30 días? & 7 & 15.9 \\
$\quad$ Sí & 35 & \\
\hline$\quad$ No & 9 & 20.5 \\
\hline Condición de Empleo & & \\
Desempleado & 9 & 20.5 \\
\hline Empleado & 35 & 79.5 \\
\hline
\end{tabular}

* Las frecuencias pueden no sumar 100\% debido a datos perdidos

* Sólo aquellas que proveyeron muestra de cabello

$\S$ Adultos que no proveyeron muestra de cabello 
Cuadro II

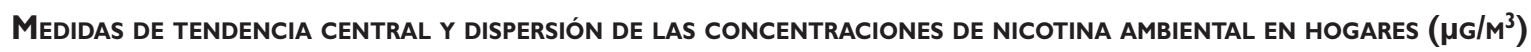
y en CAbello de mujeres no fumadoras y niños (ng/Mg). Ciudad de México, Julio de 2005

\begin{tabular}{|c|c|c|c|c|c|c|c|}
\hline Tipo de muestra & Monitores & Mediana & $Q I^{*}$ & Q3 & Min & Max & $\%$ Muestras $>C M D^{\ddagger}$ \\
\hline \multicolumn{8}{|c|}{ Nicotina ambiental - Casas $(n=4 I)$} \\
\hline \multicolumn{8}{|c|}{ Número de fumadores en casa } \\
\hline Ninguno & 8 & 0.018 & 0.007 & 0.045 & 0.001 & 0.643 & 88 \\
\hline Uno & 24 & 0.096 & 0.021 & 0.677 & 0.001 & 9.645 & 92 \\
\hline Dos o más & 9 & 0.360 & 0.059 & 0.976 & 0.006 & 6.381 & 100 \\
\hline Total & $4 I$ & 0.080 & 0.011 & 0.642 & 0.001 & 9.645 & 93 \\
\hline
\end{tabular}

Nicotina en cabello - Niños $(n=55 \xi)$

Número de fumadores en casa

\begin{tabular}{|c|c|c|c|c|c|c|c|}
\hline Ninguno & II & 0.054 & 0.054 & 0.054 & 0.054 & 0.054 & 0 \\
\hline Uno & 31 & 0.110 & 0.054 & 0.313 & 0.054 & 3.433 & 52 \\
\hline Dos o más & II & 0.253 & 0.111 & 1.015 & 0.054 & 16.130 & 82 \\
\hline Total & 53 & 0.054 & 0.054 & 0.292 & 0.054 & 16.130 & 53 \\
\hline
\end{tabular}

Nicotina en cabello - Mujeres ( $n=42)$

Número de fumadores en casa

\begin{tabular}{|c|c|c|c|c|c|c|c|}
\hline Ninguno & 7 & 0.054 & 0.054 & 0.218 & 0.054 & 0.309 & 29 \\
\hline Uno & 25 & 0.054 & 0.054 & 0.147 & 0.054 & 0.946 & 48 \\
\hline Dos o más & 10 & 0.054 & 0.054 & 0.321 & 0.054 & 1.421 & 40 \\
\hline Total & 42 & 0.054 & 0.054 & 0.192 & 0.054 & $\mathrm{I} .42 \mathrm{I}$ & 43 \\
\hline
\end{tabular}

* QI=Percentil 25, Q3=Percentil 75, Min=Mínimo, Max=Máximo, CMD= Concentración Mínima de Detección

₹ CMD = Concentración Mínima de Detección: cabello $0.054 \mathrm{ng} / \mathrm{mg}$, ambiental $0.003 \mu \mathrm{g} / \mathrm{m} 3$

$\S$ Dos niños rechazaron la toma de muestra

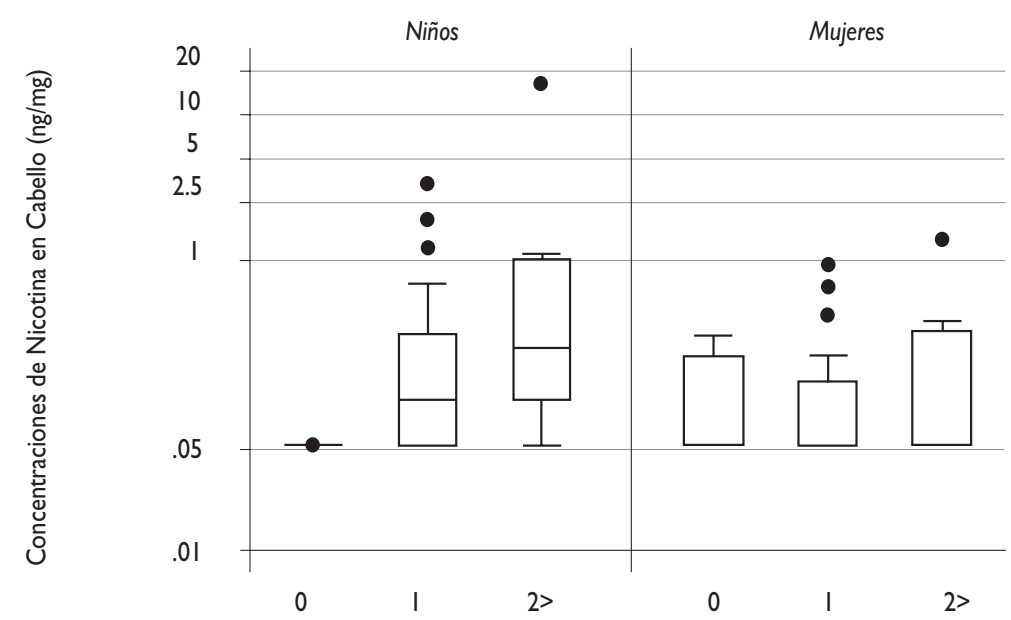

Número de fumadores en el hogar

* Las líneas horizontales dentro de los rectángulos representan la mediana; los rectángulos, el rango intercuartílico; las barras de error, los valores dentro de I.5 veces el rango intercuartílico; los puntos sólidos, las observaciones atípicas.

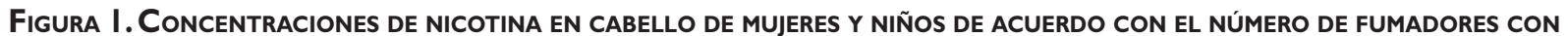
QUe conviven en el hogar. * CiUdad de México, Julio de 2005 
de $88 \%$ de los fumadores estuvo de acuerdo en que: los padres y adultos no deben fumar frente a los niños, la exposición al humo de tabaco puede causar cáncer de pulmón en no fumadores y los niños expuestos al humo de tabaco padecen más enfermedades. Setenta y cuatro por ciento de los fumadores está de acuerdo en que los lugares públicos se consideren espacios libres de humo. En contraste, por lo menos $97 \%$ de los no fumadores estuvo de acuerdo con todas las aseveraciones presentadas.

\section{Discusión}

El objetivo del presente estudio fue cuantificar los niveles de exposición involuntaria al humo de tabaco en hogares y sus habitantes no fumadores. Las concentraciones de nicotina ambiental y las de nicotina en el cabello de los niños se incrementaron de manera significativa conforme aumentó el número de fumadores en el hogar. Las concentraciones de nicotina en el cabello de las mujeres no mostraron esta tendencia. Se observó una correlación significativa $\left(r_{s}=0.49\right)$ entre las concentraciones de nicotina ambiental y las encontradas en el cabello de los niños. Esta correlación implica que $24 \%$ de la variabilidad observada en las concentraciones de nicotina en el cabello de los niños puede deberse a la exposición al humo de tabaco en el hogar.

Los hogares de la Ciudad de México presentan concentraciones ambientales de nicotina similares a las de Estados Unidos y menores a las de Taiwán. En Estados Unidos se observó una mediana de $0.01 \mu \mathrm{g} / \mathrm{m}^{3}$ en casas donde no se permit a fumar, $0.06 \mu \mathrm{g} / \mathrm{m}^{3}$ en casas donde se permit a fumar en algunos lugares y $0.66 \mu \mathrm{g} / \mathrm{m}^{3}$ en casas sin restricciones para fumar. ${ }^{21} \mathrm{En}$ Taiwán, se encontraron concentraciones de nicotina con una mediana de $0.06 \mu \mathrm{g} / \mathrm{m}^{3}$ en las casas de no fumadores y de $0.80 \mu \mathrm{g} / \mathrm{m}^{3}$ en las de fumadores. ${ }^{22}$ Las concentraciones observadas en estos pa ses concuerdan con la prevalencia de tabaquismo activo: $23.6 \%$ en Estados Unidos, $30.2 \%$ en México y $35.6 \%$ en China, sobre todo atribuible al tabaquismo masculino. ${ }^{17}$ Las concentraciones de nicotina en cabello observadas en niños de la Ciudad de México son menores que las observadas en niños de Nueva Zelanda. Niños neozelandeses con edades de 3 meses a 10 años que conviven con no fumadores presentaron una mediana menor a $0.1 \mathrm{ng} / \mathrm{mg} ; 0.275 \mathrm{ng} / \mathrm{mg}$ si conviven con un fumador; $1.46 \mathrm{ng} / \mathrm{mg}$ con dos fumadores, y $2.02 \mathrm{ng} / \mathrm{mg}$ con más de dos fumadores. ${ }^{20}$ Las concentraciones de nicotina en el cabello de niños neozelandeses se incrementaron conforme aumentó el número de fumadores en el hogar, de forma similar a lo observado en niños de la Ciudad de México. Las concentraciones de nicotina en el cabello de las mujeres del presente estudio son menores a las
Cuadro III

FACTORES DE EXPOSICIÓN Y GENERACIÓN DE HUMO DE TABACO AMBIENTAL EN NIÑOS, MUJERES NO FUMADORAS $Y$ fumadores de la Ciudad de México, JULIO DE 2005

\begin{tabular}{lcc} 
Participantes & Frecuencia & Porcentaje* $^{*}$ \\
\hline $\begin{array}{l}\text { Niños }(n=55) \\
\text { Comparte habitación con fumadores }\end{array}$ & \\
$\quad$ Sí & 22 & 40.0 \\
\hline No & 33 & 60.0 \\
\hline $\begin{array}{l}\text { Exposición a HTA en la escuela } \\
\text { Sí }\end{array}$ & 6 & \\
\hline No & 49 & 10.9 \\
\hline
\end{tabular}

Mujeres no fumadoras $\left(n=40^{\ddagger}\right)$

¿Su cónyuge fuma?

\begin{tabular}{lrl} 
Sí, pero no en casa & 9 & 22.5 \\
\hline Sí, en casa & 14 & 35.0 \\
\hline No & 12 & 30.0 \\
\hline Sin cónyuge & 5 & 12.5 \\
\hline $\begin{array}{l}\text { ¿Otros habitantes de su casa fuman? } \\
\text { Sí, pero no en casa }\end{array}$ & \\
\hline Sí, en casa & 4 & 10.0 \\
\hline No & II & 27.5 \\
\hline
\end{tabular}

¿Está expuesto a HTA en el trabajo?

\begin{tabular}{lrr} 
Sí & I & 2.5 \\
\hline No & 5 & 12.5 \\
\hline No aplica & 34 & 85.0
\end{tabular}

¿Está expuesto a HTA en el transporte?

\begin{tabular}{lcc} 
Sí & 29 & 72.5 \\
\hline No & 11 & 27.5 \\
\hline No aplica & 4 & 10.0 \\
\hline
\end{tabular}

¿Expuesto a HTA en lugares públicos?

\begin{tabular}{lrr} 
Sí & 21 & 52.5 \\
\hline No & 19 & 47.5 \\
\hline $\begin{array}{lr}\text { ¿Expuesto a HTA en restaurantes? } \\
\text { Sí }\end{array}$ & 15 & 37.5 \\
\hline No & 24 & 60.0 \\
\hline No aplica & 1 & 2.5 \\
\hline
\end{tabular}

Fumadores $(n=35)$

¿Fuma frente a los niños?

\begin{tabular}{lrr} 
Nunca & 6 & 17.1 \\
\hline A veces & 15 & 42.9 \\
\hline Frecuentemente & 8 & 22.9 \\
\hline Siempre & 6 & 17.1
\end{tabular}

¿Fuma dentro de casa?

\begin{tabular}{lrr} 
Nunca & 9 & 25.7 \\
\hline En algunos lugares & 19 & 54.3 \\
\hline En cualquier lugar & 8 & 22.9
\end{tabular}

* Las frecuencias pueden no sumar 100\% debido a datos perdidos

‡ Dos mujeres no contestaron el cuestionario 


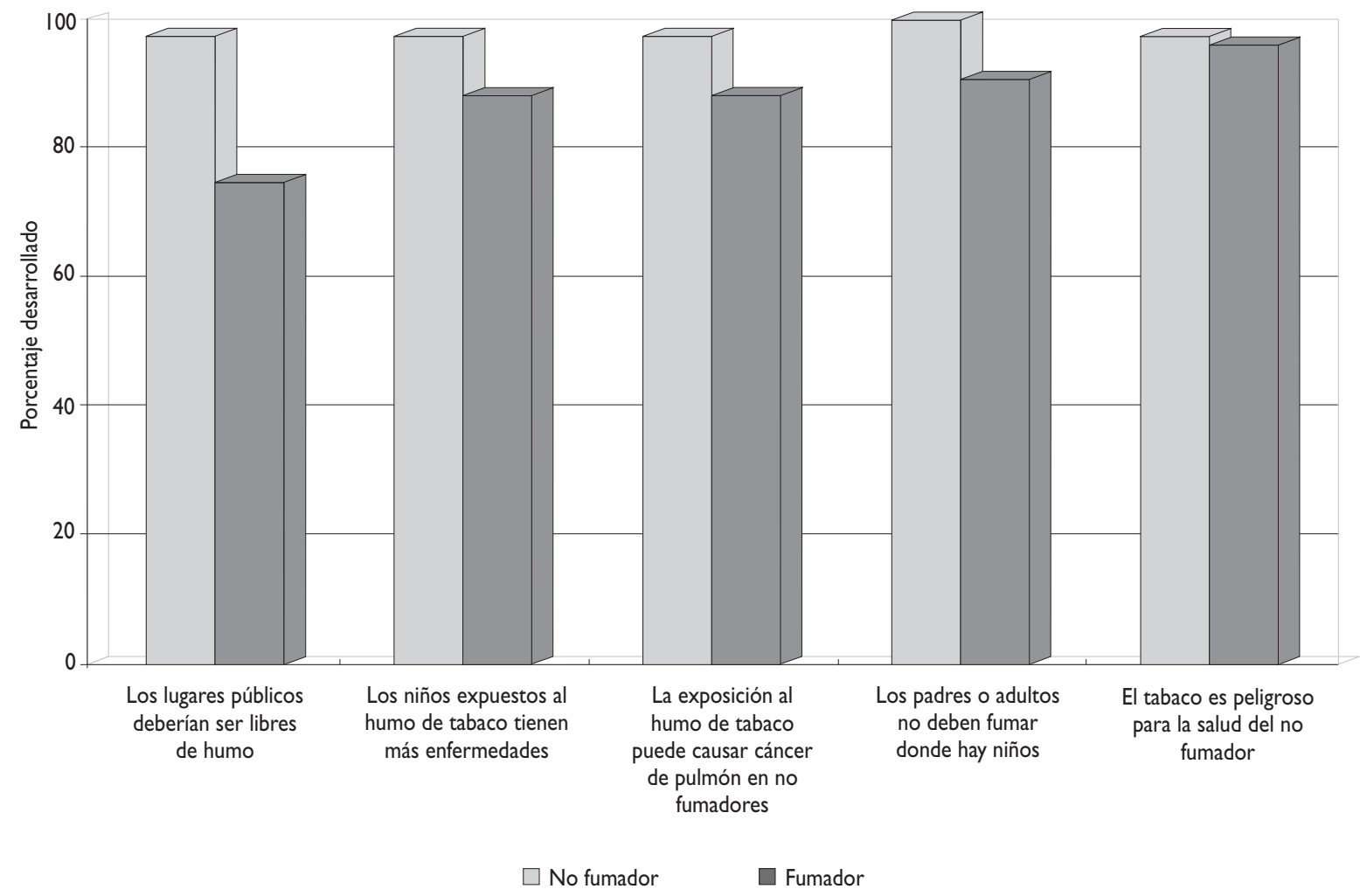

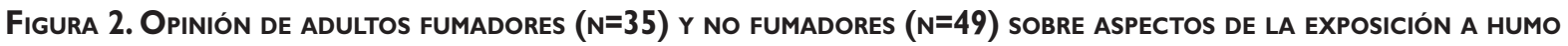
de tabaco ambiental. Ciudad de México, Julio de 2005

observadas en mujeres finlandesas y canadienses. En 389 mujeres finlandesas, aquéllas expuestas al humo de tabaco ambiental en el hogar presentaron una mediana de $2.03 \mathrm{ng} / \mathrm{mg}$, mientras que las no expuestas tuvieron una mediana de $0.86 \mathrm{ng} / \mathrm{mg} .^{23}$ En Canadá, los valores de nicotina en mujeres no expuestas al humo de tabaco ambiental presentaron una mediana de $1.2 \mathrm{ng} / \mathrm{mg}$, de $3.2 \mathrm{ng} / \mathrm{mg}$ en las expuestas y de $19.2 \mathrm{ng} / \mathrm{mg}$ en las fumadoras activas. ${ }^{24}$

La nula correlación entre el humo de tabaco ambiental y la nicotina encontrada en el cabello de las mujeres participantes en este estudio es inesperada, ya que estudios anteriores encontraron asociación entre los valores de nicotina en el cabello y el número de fumadores en casa. ${ }^{25}$ Estudios previos observaron que los tratamientos qu micos pueden disminuir el contenido de nicotina en el cabello. ${ }^{26}$ Sin embargo, en el presente estudio no se encontraron diferencias en las concentraciones de nicotina en las mujeres con y sin tratamientos en el cabello $(p=0.16)$. La influencia de otras fuentes de exposición al humo de tabaco ambiental, tales como el transporte, los restaurantes o el trabajo, también fue explorada sin observar diferencias con significancia estad stica. La selección a conveniencia de hogares impide la generalización de resultados en otros hogares. Sin embargo, más que obtener un estimado generalizable, el presente estudio provee una evaluación inicial de los grados de exposición al humo de tabaco ambiental, útil para la toma de decisiones a corto plazo y para planear estudios a mayor profundidad. La determinación de nicotina en cabello de niños ha demostrado ser sensible al número de fumadores en casa, y sugiere que puede ser útil para discriminar la exposición al humo de tabaco ambiental. Esta técnica no invasiva de evaluación es prometedora ya que posibilita la cuantificación de exposición a más largo plazo que otros biomarcadores. ${ }^{20}$ Los datos obtenidos en este estudio permiten observar el impacto que tiene el tabaquismo en el hogar sobre los niños y las mujeres no fumadoras, y as se convierte en información útil para el desarrollo de actividades preventivas a corto y largo plazo. De igual forma, el presente estudio utilizó un protocolo común a otros pa ses en desarrollo, lo cual permitirá que se generen comparaciones en el futuro y que se asienten 
las bases de un sistema internacional de vigilancia de la exposición al humo de tabaco ambiental.

Aunque las concentraciones de nicotina son menores que las observadas en pa ses como Canadá y Estados Unidos, debe recordarse que cualquier grado de exposición al humo de tabaco ambiental es nocivo, por lo que aun valores $\mathrm{m}$ nimos implican un riesgo para la salud. ${ }^{1}$ Por ello, es importante que se mantenga un sistema de evaluación a largo plazo de la exposición al humo de tabaco ambiental en los hogares que permita la adecuación constante de los programas de eliminación del consumo de tabaco y facilite la toma de decisiones. El Instituto Nacional de Salud Pública cuenta con un laboratorio anal tico de compuestos del tabaco para facilitar el mantenimiento de un sistema de vigilancia epidemiológica para la exposición al humo de tabaco (http:/ / www.insp.mx/Portal/lacot/lacot03.html). Es importante que entre las pol ticas de control al consumo de tabaco se consideren medidas para su eliminación en los hogares a través de programas integrales y de la sensibilización de los fumadores.

La información obtenida en este estudio es relevante para elevar el nivel de alerta acerca de los efectos nocivos derivados del consumo de tabaco en los hogares, que afecta a los niños en particular. El hogar es una fuente importante de exposición al humo de tabaco y debe considerarse en los esfuerzos para el control del consumo de tabaco.

\section{Agradecimientos}

Agradecemos a las siguientes personas su participación en el estudio Exposición de Mujeres y Niños a Humo de Tabaco de Segunda Mano: Georgiana Onicescu, M.S., Sungroul Kim, Ph.D, Ana Navas-Acien M.D., Ph.D, Patrick Breysse, Ph.D, y Jonathan Samet M.D., M.S. del Instituto para el Control Global del Tabaco de la Escuela de Salud Pública de la Universidad Johns Hopkins. El Flight Attendant Medical Research Institute proveyó fondos y soporte para este estudio. Agradecemos a los hogares participantes y al técnico Tito Alejandre Badillo, quien desarrolló el trabajo de campo.

Tonatiuh Barrientos-Gutiérrez cuenta con una beca Fogarty International Center Training Grant (3 D43 TW00644).

\section{Referencias}

I. US. Department of Health and Human Services. The health consequences of involuntary exposure to tobacco smoke: a report of the Surgeon General. Atlanta, GA, USA, 2006. Disponible en: http://www.surgeongeneral.gov/library/secondhandsmoke/
2. Hackshaw A, Law M,Wald N.The accumulated evidence on lung cancer and environmental tobacco smoke. Br Med J 1997;3 15:980-988.

3. Navas-Acien A, Peruga A, Breysse P, et al. Secondhand tobacco smoke in public places in Latin America, 2002-2003. JAMA 2004;29I:274I-2745. 4. Nebot M, Lopez MJ, Gorini G, et al. Environmental tobacco smoke exposure in public places of european cities. Tob Control 2005; | 4:60-63. 5.World Health Organization.WHO Framework Convention on Tobacco Control. Geneva:WHO, 2003. Disponible en: http://www.who.int/tobacco/framework/en/ 6. Goodman P,Agnew M, McCaffrey M, Paul G, Clancy L. Effects of the Irish smoking ban on respiratory health of bar workers and air quality in Dublin pubs.Am J Respir Crit Care Med 2007; 175(8):840-845.

7. Johnsson T,Tuomi T, Riuttala H, Hyvarinen M, Rothberg M, Reijula K. Environmental tobacco smoke in Finnish restaurants and bars before and after smoking restrictions were introduced.Ann Occup Hyg 2006;50:33I-34I. 8. Mulcahy M, Evans DS, Hammond SK, Repace JL, Byrne M. Secondhand smoke exposure and risk following the Irish smoking ban:An assessment of salivary cotinine concentrations in hotel workers and air nicotine levels in bars. Tob Control 2005; 14:384-388.

9. Skeer M, Land ML, Cheng DM, Siegel MB. Smoking in Boston bars before and after a 100\% smoke-free regulation: an assessment of early compliance. J Public Health Manag Pract 2004;10:50I-507.

I0. Samet JM, Yang G. Passive smoking, women and children. Women and the Tobacco Epidemic. Challenges for the 2Ist Century $200 \mathrm{I}$. Disponible en: http://www.jhsph.edu/IGTC/Resources/Chapter_ Women\%20and\%20Tobacco.pdf

II. Rohrbach LA, Howard-Pitney B, Unger JB, Dent CW, Howard KA, Cruz $\mathrm{TB}$, et al. Independent evaluation of the California tobacco control program: relationships between program exposure and outcomes, 1996-I998.Am J Public Health 2002;92:975-983.

I2. Jarvis MJ, Goddard E, Higgins V, Feyerabend C, Bryant A, Cook DG. Children's exposure to passive smoking in England since the 1980s: cotinine evidence from population surveys. Br Med J 2000;321:343-345.

13.Thomson G,Wilson N, Howden-Chapman P.Attitudes to, and knowledge of, secondhand smoke in New Zealand homes and cars. N Z Med J 2005; I I8:UI407.

14. Thomson G,Wilson N, Howden-Chapman P. Population level policy options for increasing the prevalence of smoke-free homes.J Epidemiol Community Health 2006;60:298-304.

15. World Health Organization. International consultation on environmental tobacco smoke (ETS) and child health. Consultation Report Geneva: Geneva:WHO, 1999. Disponible en: http://www.who.int/tobacco/research/ en/ets_report.pdf.

16. Ibáñez-Hernández N. Disposiciones jurídicas sobre la protección de los no fumadores en los estados de la República mexicana, en: Primer Informe Sobre El Combate Al Tabaquismo. México ante el Convenio marco para el control del tabaco. Cuernavaca, México: Instituto Nacional de Salud Pública, 2005:149-159.

17. Mackay J, Eriksen MP.The Tobacco Atlas. Geneva:World Health Organization; 2002. Disponible en: http://www.who.int/tobacco/en/atlas40.pdf

18. Hammond SK, Leaderer BP.A diffusion monitor to measure exposure to passive smoking. Environ Sci Technol 1987;21:494-497.

19. Jaakkola MS, Jaakkola JJ.Assessment of exposure to environmental tobacco smoke. Eur Respir J 1997; 10:2384-2397.

20. Berman BA,Wong GC, Bastani R, et al. Household smoking behavior and ETS exposure among children with asthma in low-income, minority households.Addict Behav 2003;28: I I I-I28.

21. Chan CC, Chen SC,Wang JD. Relationship between indoor nicotine concentrations, time-activity data, and urine cotinine-creatinine ratios in evaluating children's exposure to environmental tobacco smoke.Arch Environ Health 1995;50:230-234. 
22.Al-Delaimy WK. Hair as a biomarker for exposure to tobacco smoke. Tob Control 2002; I : 176-182.

23. Jaakkola JJK, Jaakkola N, Zahlsen K. Fetal growth and length of gestation in relation to prenatal exposure to environmental tobacco smoke assessed by hair nicotine concentration. Environ Health Perspect 200I;109:557-56I. 24. Eliopoulos C, Klein J, Phan MK, Knie B, Greenwald M, Chitayat D, et al. Hair concentrations of nicotine and cotinine in women and their newborn infants. JAMA 1994;271:621-623.
25. Nafstad P, Jaakkola J, Hagen JA, Zahlsen K, Magnus P. Hair nicotine concentrations in mothers and children in relation to parental smoking.J Expo Anal Environ Epidemiol 1997;7:235-239.

26. Pichini S,Altieri I, Pellegrini M, Pacifici R, Zuccaro P. Hair analysis for nicotine and cotinine: Evaluation of extraction procedures, hair treatments, and development of reference material. Forensic Sci Int 1997;84:243-252. 